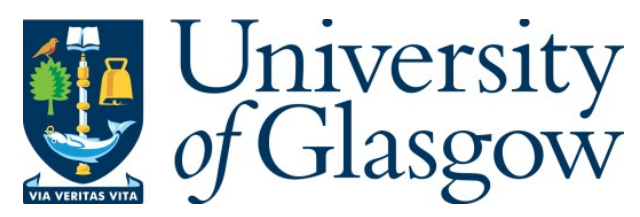

Baghramyan, H.M., Barseghyan, M.G., Laroze, D., and Kirakosyan, A.A. (2016) Influence of lateral electric field on intraband optical absorption in concentric double quantum rings. Physica E: Low-Dimensional Systems and Nanostructures, 77, pp. 81-89.

There may be differences between this version and the published version. You are advised to consult the publisher's version if you wish to cite from it.

http://eprints.gla.ac.uk/113327/

Deposited on: 14 December 2015

Enlighten - Research publications by members of the University of Glasgow http://eprints.gla.ac.uk 


\title{
Influence of lateral electric field on intraband optical absorption in concentric double quantum rings
}

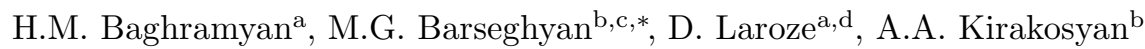 \\ ${ }^{a}$ Instituto de Alta Investigación, Universidad de Tarapacá, Casilla 7D, Arica, Chile \\ ${ }^{b}$ Department of Solid State Physics, Yerevan State University, Alex Manoogian 1, 0025 Yerevan, Armenia \\ ${ }^{c}$ National University of Architecture and Construction of Armenia, Teryan 105, 0009 Yerevan, Armenia \\ ${ }^{d}$ SUPA School of Physics and Astronomy, University of Glasgow, Glasgow G12 8QQ, United Kingdom
}

\begin{abstract}
The influence of lateral electric field on one-electron states and intraband absorption in two-dimensional concentric double quantum rings is investigated. The confining potential of the rings is modeled as a double harmonic central potential. Using the exact diagonalization technique, we calculate the dependence of the electron energy spectrum as a function of the electric field strength as well as the inner ring radius. Also, different values of confinement strength are considered. Selection rule is obtained for intraband transitions, caused by the direction of incident light polarization. The intraband absorption coefficient is calculated for different values of electric field strength, inner ring radius, confinement strength and incident light polarization direction. The combined influence of electric field strength and change of confining strength show that while the increment of the first one leads only to blueshift of absorption spectrum, the augment of the second one makes the redshift. In addition, both blueshift and redshift of the spectrum have been obtained with the enlargement of inner ring radius. Finally, we show that the absorption spectrum undergoes redshift by changing the polarization of incident light from $\mathrm{x}$ to $\mathrm{y}$ axis.
\end{abstract}

Keywords: Double quantum rings, Optical properties, Exact diagonalization method, Electric field

\section{Introduction}

Recent advances in nanoscale fabrication processes have made the construction of semiconducting nanoscopic objects with a possible wide range of geometries. Some of the most recent objects are III-V semiconducting torus shaped quantum rings (QRs) [1,2]. Like self-assembled quantum dots (QDs), QRs possess atom-like properties, making them a fair venue for potential device applications in optics, optoelectronics, and quantum computing [3-9]. At the same time QRs are a non-simply connected quantum systems - the hole in the middle provides the capability of trapping single magnetic flux and offers the exciting opportunity to observe electronic wave function phases in magneto-optical experiments. Furthermore, QRs can bind only a few electrons and holes, which gives a unique chance to study three-dimensional topological quantum effects, such as magnetization oscillations, persistent current effects, etc. [10-15].

QRs have already found their applications in optoelectronic devices, like in Ref. [4], where stacked layers of $\operatorname{In}(\mathrm{Ga}) \mathrm{As}$ on $\mathrm{GaAs}(001)$ self-assembled QRs have been studied for laser application, with stimulated multimodal emission centered at $930 \mathrm{~nm}(77 \mathrm{~K})$. Also, it is worth to

${ }^{*}$ Corresponding author. Tel. +37493504505; Fax: +37410554641

Email address: mbarsegh@ysu.am (M.G. Barseghyan) note the key importance of electric field influence in many cases. For instance, the photoluminescence and excitation of the photoluminescence spectroscopy was performed in single InGaAs self-assembled QDs and QRs embedded in a field-effect-structure device $[5,6]$. Here, the interplay between the exciton radiative recombination and the electronic carrier tunneling have been investigated in the presence of a stationary electric field and compared with a numerical calculation based on the effective mass approximation. The electronic properties of InAs/GaAs QRs and the characteristics of resonant tunnel intraband terahertz detectors with QR-active regions have done in Refs. [7, 8], where the electronic states of the QRs have been calculated and measured by the capacitance-voltage technique. Multiple layers of InAs/GaAs QRs have been used as the active region in high-performance photoconductive detectors in the range between 1-3 terahertz [9].

In the past decade, a number of works were devoted to the theoretical investigation of the influence of electric fields on the electronic and optical properties of QR structures [16-28]. For instance, in Ref. [16] the electronic states of a single QR under an applied lateral electric field is theoretically investigated for different values of the outer and inner ring radii ratio using direct matrix diagonalization method. In Ref. [17] a magnetic field perpendicular to the ring plane was used and has been shown that the electric field may suppress the Aharonov-Bohm oscillations of 
the lower energy levels. The problem of the impurity electron in QRs in the presence of a radially directed strong external electric field has been done in details in Ref. [18], where both the analytic and numerical approaches to the problem are developed.

The polarization effects of lateral electric fields and eccentricity on electronic and optical properties of QRs are discussed within the effective-mass approximation in [19]. In addition, effects of lateral electric field on the electronic states, interband optical spectra, and Aharonov-Bohm oscillations in single QRs have been investigated in Ref. [20]. The competition between carrier recombination and tunneling in QDs and QRs under the action of electric fields has been studied in Ref. [21]. Also, the effects of lateral electric field on the nonlinear optical rectification of a single QR have been investigated and the results indicate that the increase in electric field yields a redshift in peak positions of nonlinear optical rectification [22].

The simultaneous effects of lateral electric field and hydrostatic pressure on the intraband linear optical absorption coefficient have been investigated in two-dimensional GaAs/ $\mathrm{Ga}_{1-x} \mathrm{Al}_{x} \mathrm{As}$ single QR [23]. The results indicate that for fixed geometric dimensions, the hydrostatic pressure can lead to both blue- and red-shift of the intraband optical absorption spectrum, while only a blue-shift is observed as a result of electric field. The combined influences of intense laser field and static lateral electric field on oneelectron states and intraband optical absorption have been studied in two-dimensional GaAs $/ \mathrm{Ga}_{0.7} \mathrm{Al}_{0.3} \mathrm{As}$ QRs [24]. Here, the calculations have shown that by manipulating the laser field parameter and electric field strength the overlap of the electron wave functions can be altered so that the transition probability will be enhanced or suppressed on demand.

A detailed investigation concerning lateral electric field effect on single electron states in coupled quantum dot-ring (CQDR) structures has been recently performed for cases with and without an on-center hydrogenic donor impurity [25]. This study shewed that the influence of lateral electric field on energy levels strongly depends on the electron localization type. The investigations of intraband transitions in CQDR [26] demonstrated that lateral electric field changes energetic shift direction influenced by the variation of barrier thickness of the structure.

To the best of our knowledge, the influence of lateral electric field on physical properties of concentric double quantum ring (CQDR) structures have not been investigated theoretically on a large scale. The one-electron ground state in the presence of lateral electric and magnetic field as well, has only been studied in two works $[27,28]$. The aim of the present work is to study in details the effect of lateral electric field on electronic states (ground and excited) and intraband optical absorption spectrum in a two-dimensional CQDR. Furthermore, the influence of incident light polarization direction on intraband absorption spectrum would be explored. The paper is organized as follows: In Section 2 we describe the theo- retical framework. Section 3 is devoted to the results and discussions, and finally the conclusions are given in Section 4 .

\section{Theoretical Framework}

The experimental results show [2], that in double QR structures the radial sizes are around 10 times bigger than vertical sizes, which allows to consider the two-dimensional model for CDQR structure like it was done in [29-32], and consider a double parabolic central potential for the electron confinement.

$$
\begin{gathered}
V(x, y)=\frac{m \omega^{2}}{2} \min \left[\left(\sqrt{x^{2}+y^{2}}-R_{1}\right)^{2},\right. \\
\left.\left(\sqrt{x^{2}+y^{2}}-R_{2}\right)^{2}\right] .
\end{gathered}
$$

where $m$ is the electron effective mass, $\omega$ describes the strength of the confining potential, $R_{1}$ and $R_{2}$ are the inner and outer ring radii, respectively and $\min [a, b]$ means the minimum between $a$ and $b$. The width of the rings $l$ is directly related with $\omega$ via the expression $l=2 \sqrt{2 \hbar / m \omega}$. The confinement is affected by the lateral electric field directed in the $x$-axis as shown in Fig.1. Note that in the limit $R_{1} \rightarrow 0$ the potential is reduced to one of a central QD encircled with QR of a radius $R_{2}$ [25, 26, 33], while in the case of $R_{1}=R_{2}=R$ the model is reduced to a single ring structure $[1,22]$. The general form of the potential (1) has been used in Refs. [29-32, 34, 35].

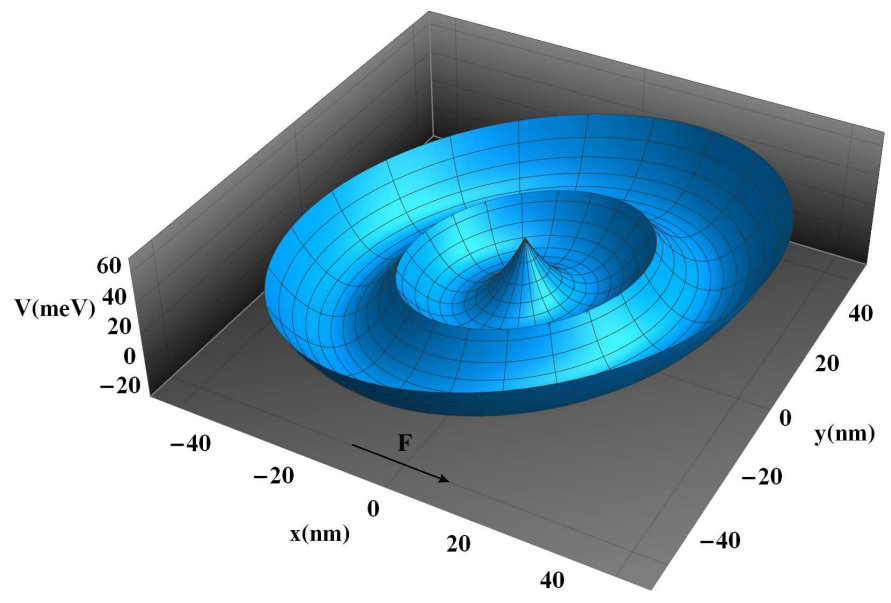

Figure 1: The confining potential for CDQR structure affected by the radial electric field. The inner and outer ring radii are correspondingly taken as $R_{1}=15 \mathrm{~nm}, R_{2}=40 \mathrm{~nm}$, confinement strength $\hbar \omega=20 \mathrm{meV}$, and electric field strength $F=5 \mathrm{kV} / \mathrm{cm}$. The direction of electric field is taken along the $+x$ axis.

The Hamiltonian of the system considering the electric field effect can be written as:

$$
\widehat{H}=-\frac{\hbar^{2}}{2 m}\left[\frac{\partial^{2}}{\partial x^{2}}+\frac{\partial^{2}}{\partial y^{2}}\right]+V(x, y)+e F x,
$$

where $e$ is the elementary charge and $F$ is the electric field strength. The electron eigenvalues $E_{N}$ and eigenfunctions 
$\Psi_{N}(x, y)$, where $N$ labels the bound electronic state in increasing order of energy, are calculated using the exact diagonalization technique $[22,24,36]$. The eigenfunctions $\Psi_{N}(x, y)$ are presented as a linear superposition of the eigenfunctions $\varphi_{n_{x}, n_{y}}(x, y)$ of the square with the side $L$ [37]:

$$
\begin{aligned}
& \varphi_{n_{x}, n_{y}}(x, y)=\frac{2}{L} \sin \left[\frac{\pi n_{x}}{L}(x-L / 2)\right] \times \\
& \sin \left[\frac{\pi n_{y}}{L}(y-L / 2)\right],
\end{aligned}
$$

where $n_{x}$ and $n_{y}$ are the quantum numbers. We remark that this technique is a useful numerical method to compute the eigenstates of quantum systems [38, 39]. In particular, it has been employed in QDs [40], in QRs [24, 41] as well as in CQDR structures [26, 42]. In addition, let us comment that in Ref. [29] finite difference scheme was used for the calculation of single electron states in the presence of magnetic field in CDQR structures and we have compared our method with it, given a very good agreement.

On the other hand, the intraband linear absorption coefficient is calculated considering Fermi's golden rule, summarized in the following expression [41, 43, 44]:

$$
\alpha(\Omega)=\frac{16 \pi^{2} \beta_{F S} \Omega}{n_{r} V} N_{i f}\left|M_{i f}\right|^{2} \delta\left(E_{f}-E_{i}-\Omega\right),
$$

where $\beta_{F S}, \Omega, n_{r}, V$ are the fine structure constant, incident photon energy, refractive index of the semiconductor and volume of the sample per CDQR, respectively. Also, $E_{i}$ and $E_{f}$ are the energies of the initial and final states and $N_{i f}=N_{i}-N_{f}$ is the difference of the electron numbers in these states. Given that we have considered a one-particle problem, $N_{i}=1$ must be taken for the ground state and $N_{f}=0$ for the excited states and the term $M_{i f}$ represents the matrix element of coordinate. In addition, to interpret the magnitude of intraband absorption strength, the oscillator strength is calculated $[45,46]$ :

$$
O_{i f}=\frac{2 m}{\hbar}\left(E_{f}-E_{i}\right)\left|M_{i f}\right|^{2}
$$

Finally, in our calculations the $\delta$-function is replaced by the Lorentzian function

$$
\delta\left(E_{f}-E_{i}-\Omega\right)=\frac{1}{\pi} \frac{\Gamma}{\left(E_{f}-E_{i}-\Omega\right)^{2}+\Gamma^{2}},
$$

where $\Gamma$ is the Lorentzian broadening parameter, which we take as $0.4 \mathrm{meV}[47]$.

\section{Results and discussion}

Calculations are performed for the following parameter values for GaAs material: electron effective mass $m=0.067 m_{0}$, where $m_{0}$ is the free-electron mass, refractive index $n_{r}=3.5$ and volume of the sample $V=$
$9.8 \times 10^{-17} \mathrm{~cm}^{3}[2]$. In Figs. 2 (a), (b) and (c) the dependencies of the ground and first two excited energy levels $(N=1,2,3)$ on the electric field strength are shown for different values of confinement strength. In the absence of electric field, the electronic states, like in any two-dimensional system with rotational symmetry, can be described by the radial quantum number $n$ and angular momentum quantum number $l$. In that system the excited states with $\pm l$ are twice degenerated, i.e. the curves for same confinement strength $\hbar \omega$ in Fig. 2 (b) and (c) start from the same point. The external electric field removes that degeneracy and mixes the states with different $n$ and $l$. All energy levels decrease with increasing electric field strength, which is a direct consequence of the lowering of confining potential bottom, i.e. a Stark effect in a parabolic quantum well [48]. Comparison of the curves in all figures shows the greater influence of the electric field on the ground state (Fig. 2 (a)), because the ground state has the deeper energy level and is affected most by the tilting effect of the confining potential. Additionally, one can observe an energy increase with the augment of confinement strength $\hbar \omega$, and that the electric field impact is greater for the smallest $\hbar \omega=10 \mathrm{meV}$, when the confinement of the potential is the weakest.

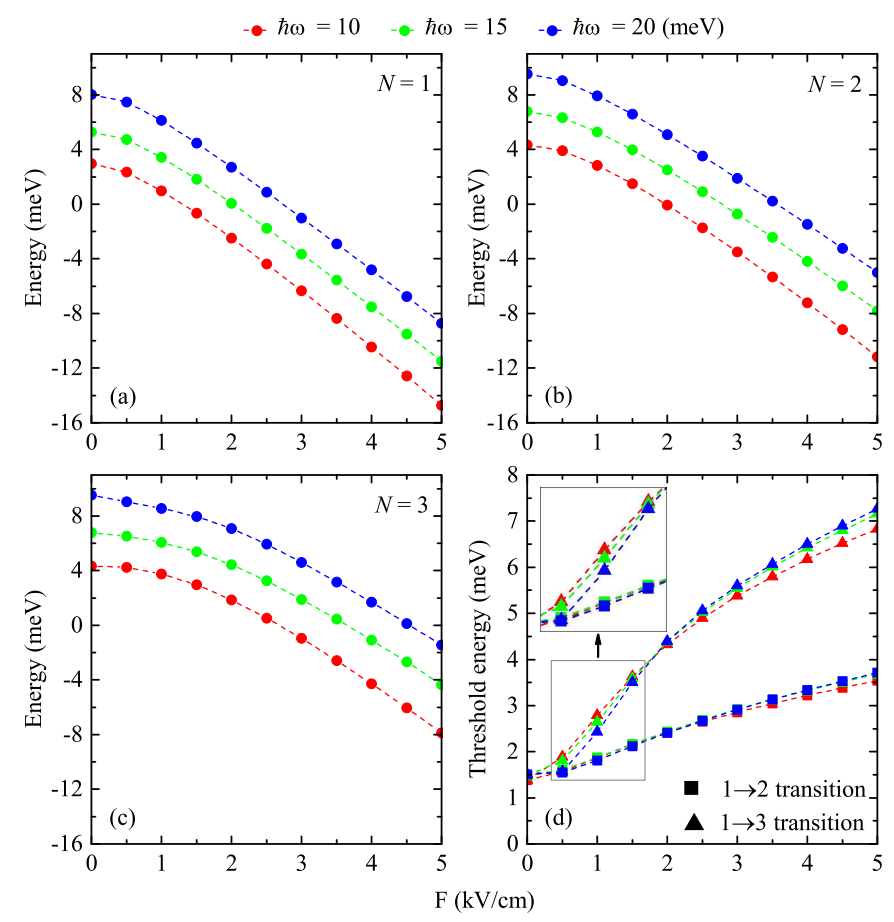

Figure 2: (color online) Dependencies of the first three states' energies (frames (a)-(c)) and the corresponding threshold energies (frame $(\mathrm{d})$ ) for the $1 \rightarrow 2$ and $1 \rightarrow 3$ transitions on electric field strength. Different confinement strengths are considered and ring radii are fixed: inner ring radius $R_{1}=15 \mathrm{~nm}$ and outer ring radius $R_{2}=40 \mathrm{~nm}$.

In Fig. 2 (d) we present the dependencies on electric field strength of the threshold energies of the $1 \rightarrow 2$ and $1 \rightarrow 3$ transitions, and the energies are the consequences 
of the results in Figs. 2 (a), (b) and (c). It is obresved that the increment of electric field increases the threshold energies of all transitions, but the strengthening of confinement, i.e. the increase of $\hbar \omega$, can result in both increase or decrease of threshold energies. The inset figure clearly shows that for $F=0.5 ; 1 ; 1.5 \mathrm{kV} / \mathrm{cm}$ an increase of $\hbar \omega$ decreases the energy, while for other values it mostly increments. Also, the influences of both electric field and confinement strength are stronger for the $1 \rightarrow 3$ transition threshold energy.

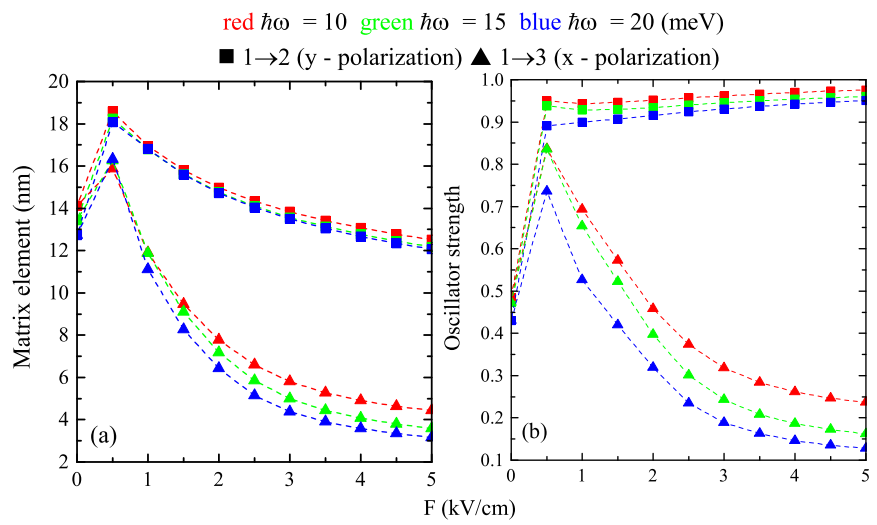

Figure 3: Dependencies of the dipole matrix element (frame (a)) and oscillator strength (frame (b)) for the allowed $1 \rightarrow 2$ and $1 \rightarrow 3$ transitions on electric field strength. Different confinement strengths and incident light polarizations are considered and ring radii are fixed: inner ring radius $R_{1}=15 \mathrm{~nm}$ and outer ring radius $R_{2}=$ $40 \mathrm{~nm}$

In Fig. 3 (a) we present the dependence on electric field strength of the dipole matrix elements for the $1 \rightarrow 2$ and $1 \rightarrow 3$ transitions, where we have used different values of confinement strengths and incident light polarization directions. The matrix element variations are interpreted with the help of the Fig. 4, where the projectional view of wave functions of considered confined states is shown for different values of electric field strength and $\hbar \omega=20 \mathrm{meV}$. It's clearly seen that, although the application of electric field removes the rotational symmetry of the potential, the eigenfunctions of system still have a certain parity under inversion with respect to the $x$-axis. In particular, the first and third states have even wave functions, while the second state has an odd wave function of $y$ variable: $\Psi_{1}(x,-y)=\Psi_{1}(x, y), \Psi_{2}(x,-y)=-\Psi_{2}(x, y)$, $\Psi_{3}(x,-y)=\Psi_{3}(x, y)$. Considering this, the selection rule is obtained, which makes the $1 \rightarrow 2$ transition feasible with $y$-polarization, whereas $1 \rightarrow 3$ transition is possible in the case of $x$-polarization of incident light. In Fig. 3 (a) one can observe that the increase of electric field strength results in the non-monotonic behavior of the matrix element. This can be explained by the corresponding overlap between investigated states $[49,50]$ (see Fig. 4). In addition, we see that for bigger $\hbar \omega$ the matrix element mostly is the smallest. The inverse variation is observed only in the case for $F=0.5 \mathrm{kV} / \mathrm{cm}$ for $x$-polarization, which is related to a different type of overlapping between the states. In Fig. 3 (b) the oscillator strength varies in accordance with Eq. 5, which is dominated by the results for the threshold energy and matrix element obtained in Fig. 2 (d) and Fig. 3 (a). It is worth to note, that in contrast with the results for matrix elements related with $1 \rightarrow 2$ transition, the corresponding oscillator strength curves show mainly an increasing behavior.

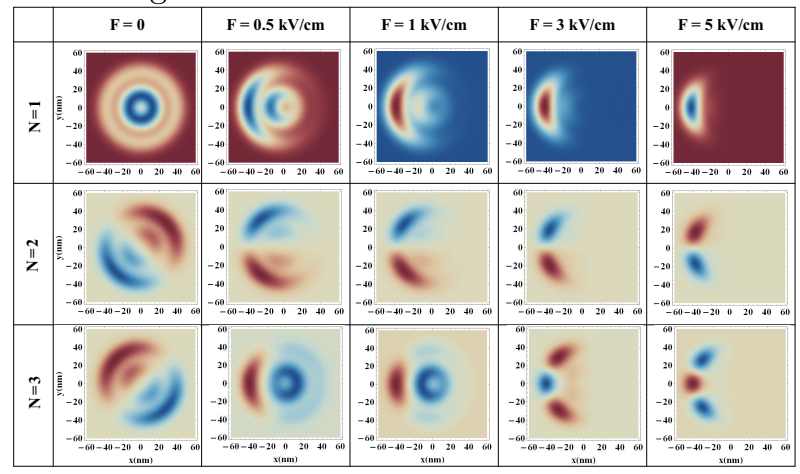

Figure 4: (color online) Projectional view of considered confined states' wave functions for different values of electric field strength and for fixed $\hbar \omega=20 \mathrm{meV}$ confinement strength.

In order to elucidate how the system's geometry modifies the energy spectrum, Fig. 5 shows the energy as a function of the inner ring radius $R_{1}$ for different strengths of electric field keeping fixed the outer radius at $R_{2}=$ $40 \mathrm{~nm}$. If $R_{1}=0$ we have dot-ring structure, with a $\mathrm{QR}$ of a radius $R_{2}$, the model of which based on Eq. 1 was studied in $[25,26,33]$. Note that the initial enlargement of $R_{1}$ makes the inner ring, thus creating wider confinement potential. Hence, the electron probability density is more spread all over the structure, than it was with dotring structure, which makes the decreasing of energies of all the states. When $R_{1}$ is increased, the system starts to modify to single ring structure with a stronger localization, and as a result of this effect an enlargement of energies is observed. When $R_{1}=R_{2}=40 \mathrm{~nm}$ we have a single ring structure modeled by parabolic confinement which was investigated in [1,22]. In addition, we observe that all energies are decreasing functions of electric field strength and that the field has a stronger impact on the ground state energy than on excited states, similar like it was shown in Fig. 2. Moreover, for higher values of the electric field strength the electronic states start to "feel" the presence of inner ring and commence to decline for bigger $R_{1}$ in all energy levels. On the other hand, Fig. 5 (d) presents the dependence on $R_{1}$ of the $1 \rightarrow 2$ and $1 \rightarrow 3$ transitions threshold energies, which like in Fig. 2 (d), are the consequences of the results in Figs. 5 (a), (b) and (c) for electron energies. 

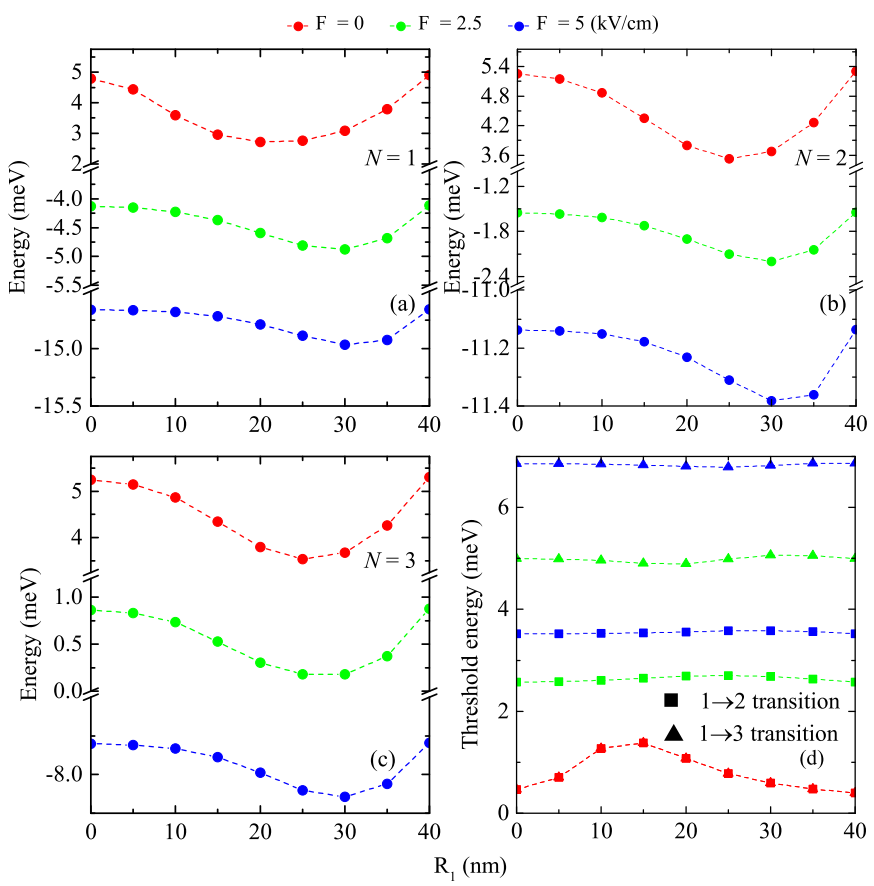

Figure 5: (color online) Dependencies of the first three states' energies (frames (a)-(c)) and the corresponding threshold energies (frame (d)) for the $1 \rightarrow 2$ and $1 \rightarrow 3$ transitions on the inner ring radius $R_{1}$ with the fixed outer one $R_{2}=40 \mathrm{~nm}$. Different electric field strengths are considered and the confinement strength is fixed by $\hbar \omega=10 \mathrm{meV}$.

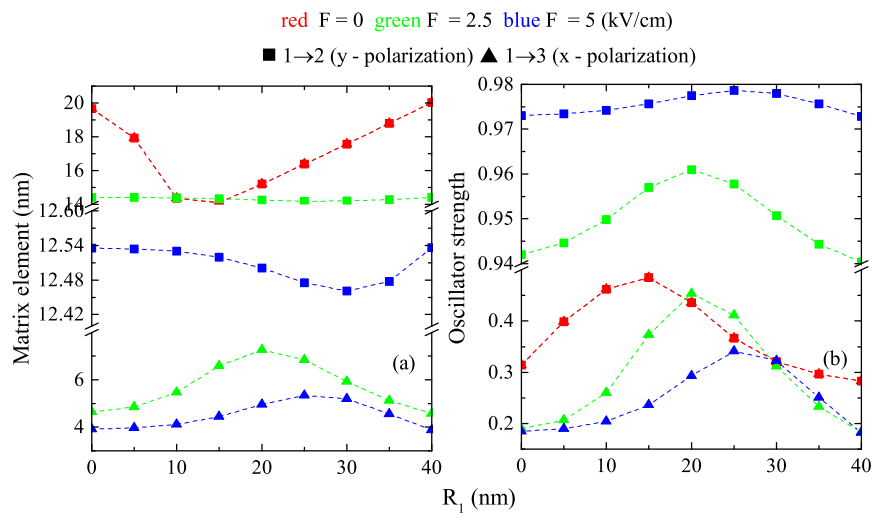

Figure 6: Dependencies of the dipole matrix element (frame (a)) and oscillator strength (frame (b)) for the allowed $1 \rightarrow 2$ and $1 \rightarrow$ 3 transitions on the inner ring radius $R_{1}$ with the fixed outer one $R_{2}=40 \mathrm{~nm}$. Different electric field strengths are considered and the confinement strength is fixed by $\hbar \omega=10 \mathrm{meV}$.

In Fig. 6 (a) we preset the dipole matrix elements dependence on the inner ring radius $R_{1}$ for the $1 \rightarrow 2$ and $1 \rightarrow 3$ transitions, considering different values of electric field strength and incident light polarization direction. Almost all the curves show the non-monotonic behavior on $R_{1}$ variations as a result of structural change from dotring to double ring structure, which have different shapes of probability density distribution. It must be noted that those variations are more explicit in the absence of the field, which is quite understandable considering that elec- tric field has a power to destroy the initial rotational symmetry of confining potential, like it was mentioned in the discussion of Fig. 3 (a). Also we have the expected result of the same matrix elements for both transitions in the absence of electric field, and that in the presence of the electric field the matrix elements of $1 \rightarrow 2$ y-polarized transition are bigger than the ones for x-polarized $1 \rightarrow 3$ transition (the similar result was obtained in Fig. 3 (a)). The results for oscillator strength in Fig. 6 (b) will help us to understand the changes of absorption maxima that we will present in the upcoming figures.

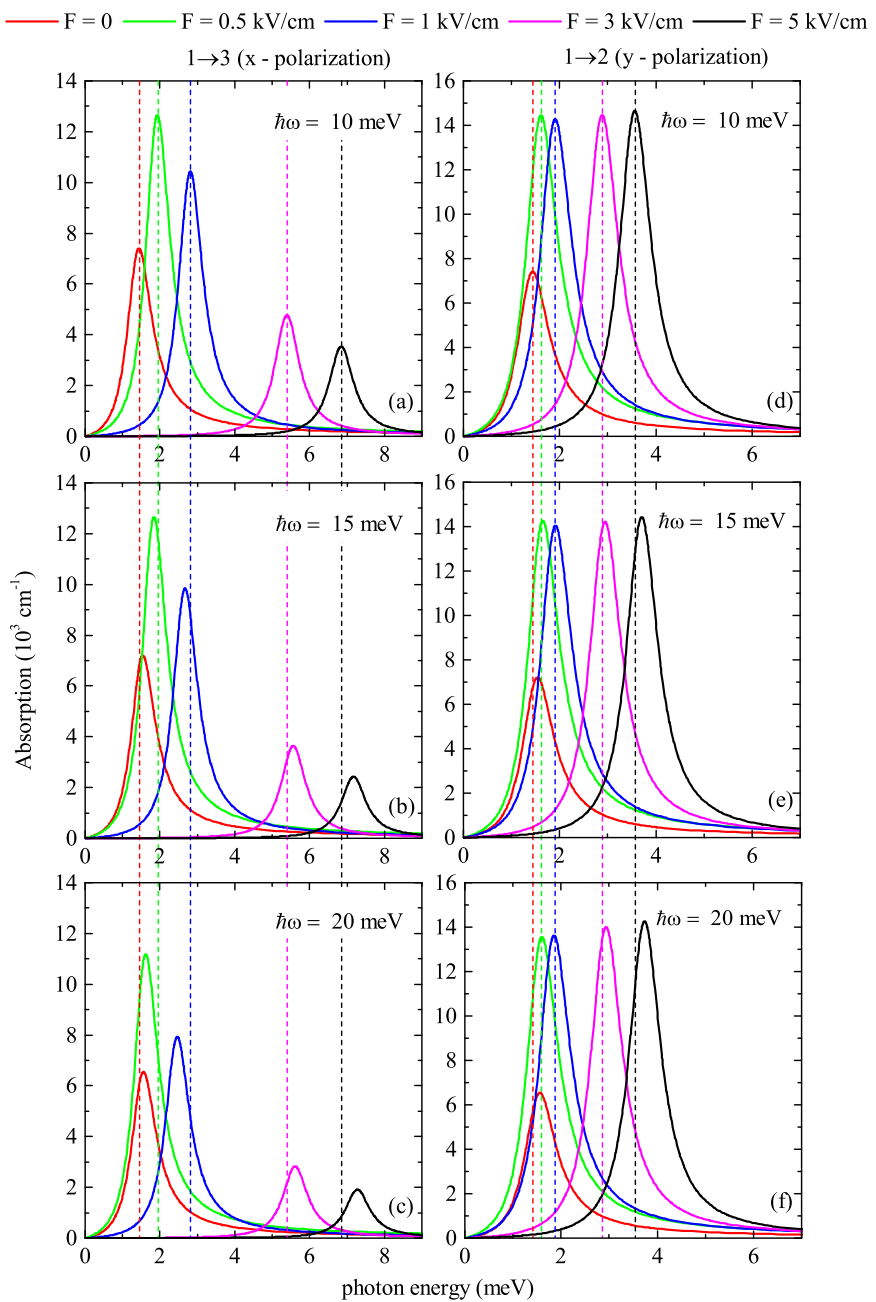

Figure 7: Dependencies of intraband absorption coefficient on the incident photon energy for different values of electric field strength and confinement strength: $x$-polarization of the incident light is taken for $1 \rightarrow 3$ transition (frames (a)-(c)) and $y$-polarization for $1 \rightarrow 2$ transition (frames (d)-(f)). Ring radii are fixed: $R_{1}=15 \mathrm{~nm}$ and $R_{2}=40 \mathrm{~nm}$.

The intraband linear absorption coefficient dependence on the incident photon energy for different strengths of the electric field, confinement strength and light polarization direction is shown in the Fig. 7 . As a consequence of threshold energy dependencies in the Fig. 2 (d), we observe a blueshift in the absorption spectrum with the increase of electric field in all the figures. In Figs. 7 (a)- 
(c) the increase of $\hbar \omega$ forces all the curves to undergo the blueshift, except the green and blue curves, which correspond to $F=0.5 \mathrm{kV} / \mathrm{cm}$ and $F=1 \mathrm{kV} / \mathrm{cm}$ respectively. These curves show a redshift in absorption spectrum. In Figs. 7 (d)-(f) the blueshift of the spectrum dominates.

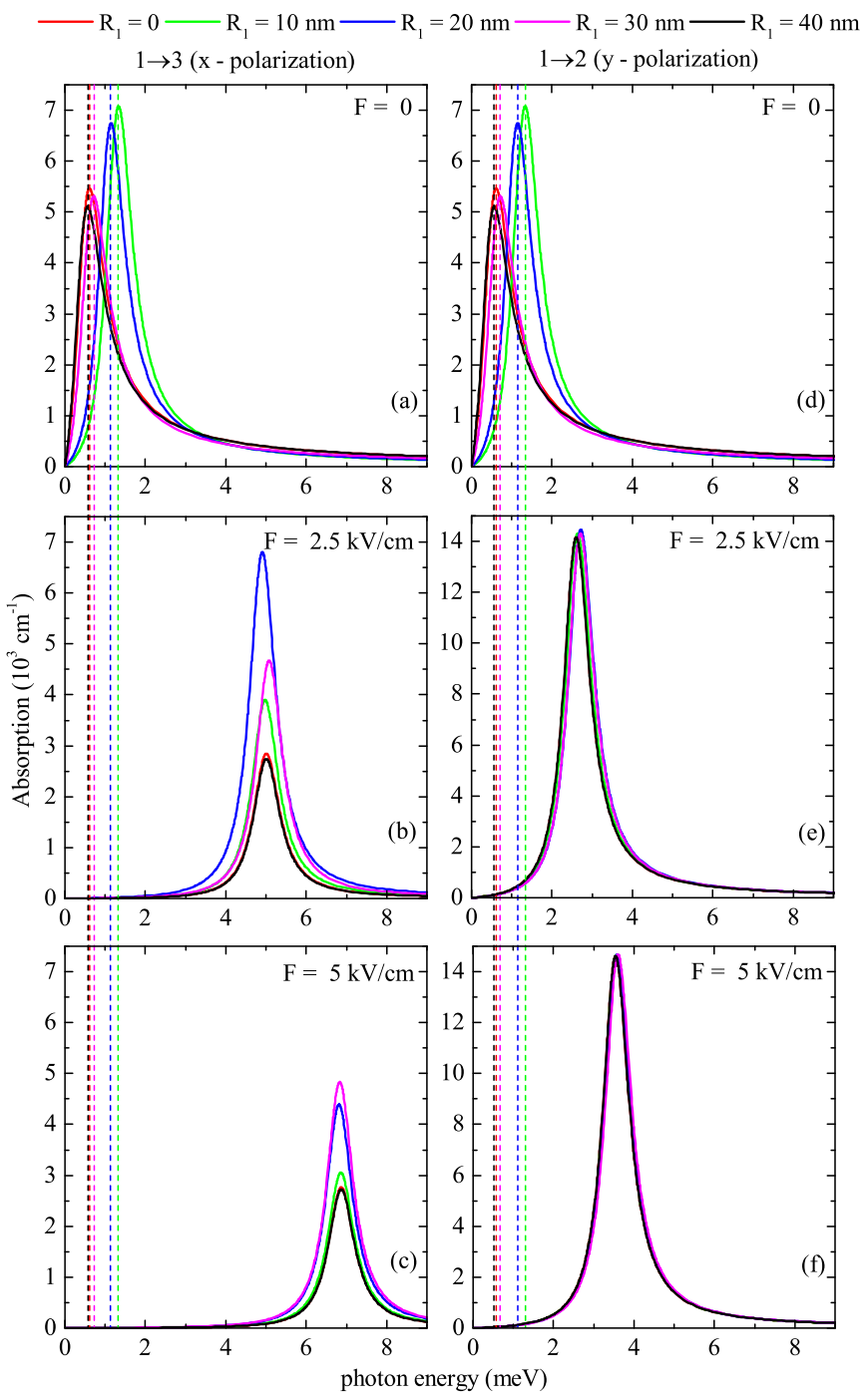

Figure 8: Dependencies of intraband absorption coefficient on the incident photon energy for different values of inner ring radius $R_{1}$ (outer one is fixed as $R_{2}=40 \mathrm{~nm}$ ) and electric field strength: $x$ polarization of the incident light is taken for $1 \rightarrow 3$ transition (frames 8 (a)-(c)) and $y$-polarization for $1 \rightarrow 2$ transition (frames (d)-(f)), and the confinement strength is fixed by $\hbar \omega=10 \mathrm{meV}$.

Furthermore, if we compare the absorption curves for the same electric field strength and confinement strength but different polarization, we notice that a change from $x$ to $y$ axis direction will yield a redshift of the absorption spectrum considering a non-zero electric field. In addition, we observe an increase and decrease of absorption maxima with increasing electric field strength. The decrease of absorption maxima is seen with increasing $\hbar \omega$ or with the change of $x$-polarization to $y$-polarization (according to the Fig. $3(\mathrm{~b})$ ), and the $1 \rightarrow 3$ transition absorption max- imum is smaller than that of the $1 \rightarrow 2$ transition. The variation of absorption maximum is linearly controlled by the corresponding change in oscillator strength as shown in Fig. 3 (b), like it is mentioned during the discussion of Eq.(5).

Finally in the Fig. 8 we have the intraband linear absorption coefficient dependence on the incident photon energy for different values of inner radius $R_{1}$, strength of the electric field and light polarization direction. With the increase of $R_{1}$ both blueshift and redshift of absorption spectrum have been obtained according to the results in Fig. 5 (d) for threshold energy, but the results are more clear for transitions in the case of zero electric field. The increment of electric field strength creates the blueshift of the spectrum and like in previous figure, change of the polarization direction from $x$ axis to $y$, makes the redshift. Added to it, in accordance with the results for oscillator strength in Fig. 6 (b), the maximum of absorption spectrum undergoes the non-monotonic shifts with the increase of $R_{1}$ and, in the presence of electric field, definitely increases while changing the polarization direction from $\mathrm{x}$ axis to y. Meanwhile, the augment of electric field strength bring the blueshift of the spectrum, similar to the phenomenon obtained in Fig. 7.

\section{Conclusions}

In this paper we have studied the influence of lateral electric field on one-electron states and intraband absorption in concentric double quantum rings. We have found that electric field influence induces a decrease in the energies of the ground and first two excited states, while the strengthening of the confinement potential, by increasing the confinement strength lead to the augment of energies and the enlargement of inner ring radius creates non-monotonic variation of energies. The full analysis of electric field influence on one-electron state wave-function distributions has been performed. With our analysis a selection rule is found dependent on incident light polarization. A blueshift in absorption spectrum is obtained by increasing the electric field, although a confinement strength and inner ring radius increment may lead to redshift as well. In addition, a change in light polarization from $x$ axis to $y$-axis creates a redshift in the absorption spectrum. Furthermore, the absorption maximum is bigger for $y$-polarized light and for smaller confinement strengths, while it changes in a non-monotonic way if the electric field and inner ring radius increases. We consider that the influence of both electric field and light polarization on intraband spectrum offers attractive new strategies to fine-tune optoelectronic devices based on quantum rings, and moreover, to better understand quantum information phenomena in coupled nanostructures. 


\section{Acknowledgements}

HMB acknowledges financial support from CONICYTFONDECYT Postdoctral program fellowship under grant 3150109. The work of MGB was supported by the Armenian State Committee of Science (Project no. 13YR1C0012). DL acknowledges partial financial support from Basal Program Center for Development of Nanoscience and Nanotechnology (CEDENNA) and UTA-project 875012.

[1] A. Lorke, R.J. Luyken, A.O. Govorov, J.P. Kotthaus, J.M. Garcia, P.M. Petroff, Phys. Rev. Lett. 84 (2000) 2223.

[2] T. Mano, T. Kuroda, S. Sanguinetti, T. Ochiai, T. Tateno, J. Kim, T. Noda, M. Kawabe, K. Sakoda, G. Kido, N. Koguchi, Nano Lett. 5 (2005) 425.

[3] T. Chakraborty, Adv. in Solid State Phys. 43, (2003) 79.

[4] F. Suárez, D. Granados, M.L. Dotor, J.M. García, Nanotechnology 15 (2004) S126.

[5] B. Alén, J. Martínez-Pastor, D. Granados, J.M. García, Phys. Rev. B 72 (2005) 155331.

[6] B. Alén, J. Bosch, D. Granados, J. Martínez-Pastor, J.M. García, L. González, Phys. Rev. B 75 (2007) 045319.

[7] G. Huang, W. Guo, P. Bhattacharya, G. Ariyawansa, A.G.U. Perera, Appl. Phys. Lett. 94 (2009) 101115.

[8] Jong-H. Dai, Jheng-Han Lee, Yi-Lung Lin, Si-Chen Lee, Jap. Jour. Appl. Phys. 47 (2008) 2924.

[9] S. Bhowmick, G. Huang, W. Guo, C.S. Lee, P. Bhattacharya, G. Ariyawansa, A.G.U. Perera, Appl. Phys. Lett. 96 (2010) 231103.

[10] Y. Aharonov, D. Bohm, Phys. Rev. 115 (1959) 485.

[11] P. Pietiläinen, T. Chakraborty, Solid State Commun. 87, (1993) 809 .

[12] K. Niemelä, P. Pietiläinen, P. Hyvänen, T. Chakraborty, Europhys. Lett. 36, (1996) 533.

[13] A. Fuhrer, S. Lüscher, T. Ihn, T. Heinzel, K. Ensslin, W. Wegscheider, M. Bichler, Nature 413 (2001) 822.

[14] A. Mühle, W. Wegscheider, R.J. Haug, Appl. Phys. Lett. 91 (2007) 133116.

[15] D.S.L. Abergel, V.M. Apalkov, T. Chakraborty, Phys. Rev. B 78 (2008) 193405.

[16] J.M. Llorens, C. Trallero-Giner, A. García-Cristóbal, A. Cantarero, Phys. Rev. B 64 (2001) 035309.

[17] A. Bruno-Alfonso, A. Latgé, Phys. Rev. B 71 (2005) 125312.

[18] B.S. Monozon, M.V. Ivanov, P. Schmelcher, Phys. Rev. B 70 (2004) 205336.

[19] L.A. Lavenére-Wanderley, A. Bruno-Alfonso, A. Latgé, J. Phys.: Condens. Matter 14 (2002) 259.

[20] Z. Barticevic, G. Fuster, M. Pacheco, Phys. Rev. B 65 (2002) 193307.

[21] J. Bosch, B. Alén, J. Martínez-Pastor, D. Granados, J.M. García, L. González, Superlatt. Microstruct. 43 (2008) 582.

[22] W. Xie, Physica B 443 (2014) 60.

[23] A.Kh. Manaselyan, M.G. Barseghyan, A.A. Kirakosyan, D. Laroze, C.A. Duque, Physica E 60 (2014) 95.

[24] A. Radu, A.A. Kirakosyan, D. Laroze, M.G. Barseghyan, Semicond. Sci. Technol. 30 (2015) 045006.

[25] M.G. Barseghyan, Physica E 69 (2015) 219.

[26] M.G. Barseghyan, H.M. Baghramyan, D. Laroze, J. Bragard, A.A. Kirakosyan, Physica E 74 (2015) 421.

[27] A. Shahbandari, M.A. Yeranosyan, A.L. Vartanian, Superlatt. Microstruct. 57 (2013) 85.

[28] M.A. Yeranosyan, A.L. Vartanian, A. Shahbandari, A.A. Kirakosyan, Physica B 456 (2015) 365.

[29] B. Szafran, F.M. Peeters, Phys. Rev. B 72 (2005) 155316.

[30] F. Malet, M. Pi, M. Barranco, E. Lipparini, Ll. Serra, Phys. Rev. B 74 (2006) 193309.

[31] L. Colletti, F. Malet, M. Pi, F. Pederiva, Phys. Rev. B 79 (2009) 125315.
[32] J.M. Escartín, F. Malet, M. Barranco, M. Pi, Physica E 42 (2010) 841.

[33] B. Szafran, F.M. Peeters, S. Bednarek, Phys. Rev. B 70 (2005) 125310.

[34] A. Wensauer, O. Steffens, M. Suhrke, U. Rössler, Phys. Rev. B 62 (2000) 2605.

[35] T. Chwiej, B. Szafran, Phys. Rev. B 78 (2008) 245306.

[36] G.A. Evans, J.M. Blackledge, P.D. Yardley, Numerical methods for partial differential equations (Springer, London, 1990).

[37] S. Gangopadhyay, B.R. Nag, Phys. Stat. Sol. (b) 195 (1996) 123.

[38] P. Michler (Editor), Single Quantum Dots: Fundamentals, Applications and New Concepts (Springer, Berlin, 2003)

[39] H. Fehske, R. Schneider, and A. Weiße, (Editors) Computational Many-Particle Physics (Springer, Heidelberg, 2008).

[40] A. Tiutiunnyk, V. Tulupenko, M.E. Mora-Ramos, E. Kasapoglu, F. Ungan, H. Sari, I. Sökmen, C.A. Duque, Physica E 60 (2014) 127.

[41] A. Radu, A.A. Kirakosyan, D. Laroze, H.M. Baghramyan, M.G. Barseghyan, J. Appl. Phys. 116 (2014) 093101.

[42] J.D. Correa, M.E. Mora-Ramos, C.A. Duque, Physica B: Condens. Matter 472 (2015) 25.

[43] H. Taş, M. Şahin, J. Appl. Phys. 112 (2012) 053717.

[44] S.L. Chuang, Physics of Photonic Devices, 2nd Edition, Wiley Series in Pure and Applied Optics, Wiley, New York, 1995.

[45] R.W. Boyd, Nonlinear Optics, 3rd Edition, Academic Press, San Diego, 2003.

[46] S. Liang, W. Xie, H.A. Sarkisyan, A.V. Meliksetyan, H. Shen, J.Phys.: Condens. Matter 23 (2011) 415302.

[47] M. Sugawara (Ed.), Theoretical bases of the Optical Properties of Semiconductor Quantum Nano-Structures, vol, 60 of Semicond. and Semimet., Elsevier, New York, 1999.

[48] S.L. Chuang, D. Ahn, J. Appl. Phys. 65 (1989) 2822.

[49] R.C. Iotti, L.C. Andreani, Phys. Rev. B 56 (1997) 3922.

[50] F. Koç, M. Şahin, J. Appl. Phys. 115 (2014) 193701. 